\title{
Mitteilungen der agswn
}

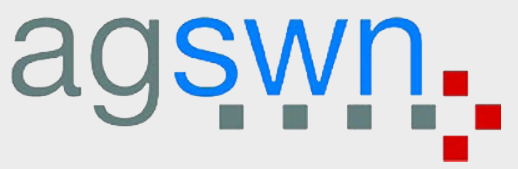

Notfall Rettungsmed 2020 23:240

https://doi.org/10.1007/s10049-020-00705-8

(c) Springer Medizin Verlag GmbH, ein Teil von Springer Nature 2020
Arbeitsgemeinschaft Südwestdeutscher Notärzte e.V. (agswn)

c/o Geschäftsstelle

Rita-Maiburg-Str. 2, 70794 Filderstadt

geschaeftsstelle@agswn.de

www.agswn.de

\section{Redaktion}

Prof. Dr. med. Matthias Fischer (V.i.S.d.P.)

Priv.-Doz. Dr. med. H. Genzwürker
Liebe Kolleginnen und Kollegen, sehr geehrte Damen und Herren,

aufgrund der zunehmenden Verbreitung des Coronavirus SARS-CoV-2 sowie der hohen Einbindung gerade vieler notfallmedizinisch Engagierter sahen wir uns leider gezwungen, die 35 . notfallmedizinische Jahrestagung der agswn am 13./14.03.2020 abzusagen.

Führend für diese Entscheidung war die Tatsache, dass es sich bei den Teilnehmerinnen und Teilnehmern überwiegend um notärztliches und rettungsdienstliches Personal handelt, so dass die Gefahr von Quaran- tänemaßnahmen in der Folge unserer Tagung zu erheblichen Einschränkungen der notfallmedizinischen Versorgung im Südwesten und darüber hinaus bestand.

Wir haben uns deshalb schweren Herzens zu diesem Schritt entschieden und bitten um Ihr Verständnis für dieses Vorgehen.

Wir freuen uns auf Sie nächstes Jahr am 12./13.03.2021 in Baden-Baden!

\section{Ihre}

Arbeitsgemeinschaft Südwestdeutscher Notärzte e.V. agswn

\section{Was bietet Ihnen die Mitgliedschaft?}

Als Mitglied der Interessenvertretung der Notärztinnen und Notärzte in Baden-Württemberg, Rheinland-Pfalz und dem Saarland erhalten Sie für einen jährlichen Mitgliedsbeitrag von EUR 70,00 unter anderem die Zeitschrift "Notfall + Rettungsmedizin" mit 8 Ausgaben pro Jahr. Zusätzlich ist die Teilnahme an der Jahrestagung im März in Baden-Baden für Mitglieder kostenlos. Darüber hinaus können Sie auf Inhalte im Mitgliederbereich der Homepage zugreifen. Für Studenten und Rettungsdienstpersonal bietet die agswn e.V. für EUR 35,00 im Jahr eine außerordentliche Mitgliedschaft mit allen vorgenannten Leistungen.

Seit mehr als 30 Jahren setzen wir uns gemeinsam mit mittlerweile um die 2000 Mitgliedern erfolgreich für die Weiterentwicklung des Notarztdienstes und der notfallmedizinischen Versorgung der Bevölkerung unserer drei Mitgliedsländer ein. Kennen Sie Kolleginnen und Kollegen, denen Sie die Mitgliedschaft empfehlen möchten? Die Anmeldung ist direkt auf der Homepage möglich.

Ihre Ansprechpartner für Fragen rund um die Notfallmedizin in den Landesverbänden und den Regionen finden Sie auf der agswn-Homepage unter www.agswn.de/vorstand und www.agswn.de/regionalbeauftragte. Auch die Geschäftsstelle steht Ihnen für Anfragen zur Verfügung.

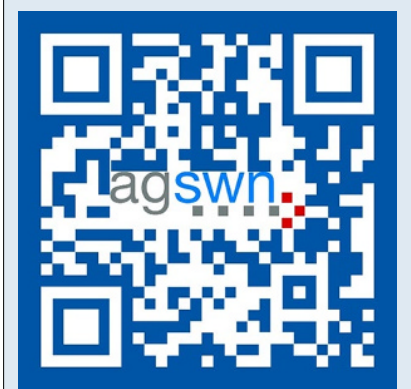

Auf der Homepage der agswn können Sie sich auch mit Ihrer E-Mail-Adresse für den Newsletter anmelden.

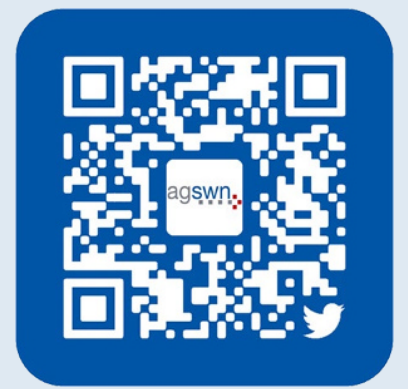

Bei Twitter informieren wir Sie unter agswn_ev mit Kurzmeldungen aus dem agswn-Land. 